\title{
A combination of tryptophan, Satureja montana, Tribulus terrestris, Phyllanthus emblica extracts is able to improve sexual quality of life in patient with premature ejaculation
}

\author{
Salvatore Sansalone ${ }^{1}$, Giorgio Ivan Russo ${ }^{2}$, Nicola Mondaini ${ }^{3}$, Francesco Cantiello ${ }^{4}$, \\ Gabriele Antonini ${ }^{5}$, Tommaso Cai ${ }^{6}$ \\ ${ }^{1}$ Department of Experimental Medicine and Surgery, University Tor Vergata, Rome, Italy; \\ ${ }^{2}$ Department of Urology, University of Catania, Catania, Italy; \\ ${ }^{3}$ Urologic Unit, Santa Maria Annunziata Hospital, Bagno a Ripoli, Florence, Italy; \\ ${ }^{4}$ Urology Unit, Magna Graecia University of Catanzaro, Italy; \\ ${ }^{5}$ Department of Urology, "Sapienza" Rome University, Rome, Italy; \\ ${ }^{6}$ Department of Urology, Santa Chiara Regional Hospital, Trento, Italy.
}

\begin{abstract}
Summary Objective: The management of patient affected by premature ejaculation (PE) is nowadays not highly satisfactory. Here, we aimed to evaluate the tolerability and efficacy of a combination of tryptophan, Satureja montana, Tribulus terrestris, Phyllanthus emblica extracts in order to improve sexual quality of life in patients with premature ejaculation.

Materials and methods: All patients attending to 5 urological centers from January 2015 to March 2015, due to premature ejaculation were enrolled in this study. At the enrolment visit, all subjects underwent self-administered IIEF-5, Male Sexual Health Questionnaire-Ejaculation Disorder (MSHQEjD), PEDT and IELTS (calculated as mean from that perceived by partner and that perceived by patient) and underwent urological visit and laboratory examinations. All patients received one tablet per day of a combination of tryptophan, Satureja montana, Tribulus terrestris, Phyllanthus emblica extracts for 3 months (Group A). After 3 months all patients underwent follow-up visit with the same investigations that have been carried out in the enrolment visit. The results were compared with a cohort of patients enrolled in the same period in another urological center and considered as a control group (Group B). All patients in the control group underwent counseling and sexual behavioral treatment without any pharmacological compound.

Results: At the follow-up analysis, significant changes in terms of IELT in the Group A (mean difference: $31.90 ; p<$ 0.05 ) at 3 months and versus Group $B$ at the intergroup analysis (mean difference: $30.30 ; p<0.05$ ) were reported. In the group A, significant differences from baseline to last follow-up were observed relative to IIEF-5 (mean difference: 1.04; $p<0.05$ ), PEDT (mean difference: $-2.57 ; p<0.05$ ) and FSH (mean difference: $-16.46 ; p<0.05)$.

Conclusion: In conclusion, patients affected by PE may significantly benefit from oral therapy with a combination of tryptophan, Satureja montana, Tribulus terrestris, Phyllanthus emblica extracts in terms of IELT and PEDT scores improvement.
\end{abstract}

KEY WORDS: Premature ejaculation; Erectile dysfunction; Tryptophan; Satureja montana; Tribulus terrestris; Phyllanthus emblica.

Submitted 9 November 2015; Accepted 4 December 2015

\section{INTRODUCTION}

Over the past 30 years, the premature ejaculation (PE) treatment paradigm, which previously limited to behavioral psychotherapy, has expanded to include drug treatment (1). Dapoxetine (Priligy, Johnson \& Johnson, New Brunswick, NJ) is the first and the only product licensed for the treatment of PE in men aged 18-64 years, and it belongs to a class of drugs called selective serotonin reuptake inhibitors (SSRIs), which are frequently used to treat depression (2-4). Across trials, receiving dapoxetine 30 and $60 \mathrm{mg}$ were well tolerated with a low incidence of adverse events (AEs) (2). The most common AEs were those typically accompanied with SSRIs agents, and the symptoms were mild (2). Furthermore, although treatment with marketed SSRIs had been associated with sexual dysfunctions and suicide attempts, there were no reports on sexual dysfunctions or suicide events as well as suicide attempts in these 5 RCTs (5-6). However, there were limited available data about using dapoxetine for a long time. Phytotherapeutics should be an interesting option because of their generally low side effects and high acceptance by the patients. We focused our attention on some compounds that may be useful for the treatment of PE. Tryptophan is an interesting phytotherapeutic compound. 5-Hydroxytryptophan (5-HTP) is the intermediate metabolite of the essential amino acid L-tryptophan in the biosynthesis of serotonin (7-8). The role of serotonin in the ejaculation control is well known and the well demonstrated (9-10). Moreover, 5-HTP is well absorbed from an oral dose, with about $70 \%$ was found in the bloodstream (11). Furthermore, 5-HT showed a good pharmacological profile with good efficacy and appeared to be better tolerated than SSRIs (12). On the other hand, Satureja montana (winter savory) is a medicinal plant traditionally used to treat different disorders including male sexual dysfunction (13). Recently, Zavatti et al. found that Satureja montana is able to delay ejaculation latency without exerting any negative effect on the other parameters of sexual behavior and without exerting a sedative effect (13). Finally, the role of Phyllanthus emblica. Chebulagic 
acid, the major constituent of Phyllanthus emblica, is a benzopyran tannin compound with various kinds of medicinal potentials including improvement of male sexual dysfunction (14-15). In fact, Chebulagic acid is able to inhibit Rho-kinase 2 and may be useful in management of erectile dysfunction (14). Here, we aim to evaluate the tolerability and efficacy of a combination of tryptophan, Satureja montana, Tribulus terrestris, Phyllanthus emblica extracts (EIACUMEV ${ }^{\circledR}$ ) in order to improve sexual quality of life in patients with premature ejaculation.

\section{Patients ANd Methods}

\section{Study design}

All patients attending to 5 urological centers from January 2015 to March 2015, due to premature ejaculation were enrolled in this study. At the enrolment visit, all subjects underwent self-administered IIEF-5, Male Sexual Health Questionnaire-Ejaculation Disorder (MSHQ-EjD), PEDT and IELTS and underwent urological visit and laboratory examinations. The IELT has been calculated as mean from that perceived by partner (PPIELT) and that perceived by patient (IELT). All patients underwent preliminary assessment including a detailed medical and sexual history to evaluate the presence of underlined risk factors such as infections or diabetes mellitus. All patients received one tablet per day of a combination of tryptophan, Satureja montana, Tribulus terrestris, Phyllanthus emblica extracts for 3 months (Group A). After 3 months all patients underwent follow-up visit with the same investigations that have been carried out in the enrolment visit. The results were compared with a cohort of patients enrolled in the same period in another urological center and considered as a control group (Group B). All patients in the control group underwent counseling and sexual behavioral treatment without any pharmacological compound. All subjects gave written informed consent before entering the study, which was conducted in accordance with the Declaration of Helsinki.

\section{Inclusion and exclusion criteria}

The primary inclusion criteria were age $\geq 18$ years of age and in a stable monogamous relationship for $\geq 6$ months were eligible if they met the DSM-IV-TR criteria for PE for $\geq 6$ months, indicated at least moderate PE-related distress or interpersonal difficulty, and reported an IELT of $\leq 2$ min in $\geq 75 \%$ of evaluable events during a 4 -wk screening/baseline period. Exclusion criteria were as follows: any medical treatment for sexual dysfunction before or during the study, congenital or acquired penile curvature or chordee with hypospadias, age $>75$ years, hypogonadism (total testosterone level $<8 \mathrm{nmol}$ or serum testosterone in the range of $8-11 \mathrm{nM}$ and free testosterone $<220 \mathrm{pmol}$, assessed at least on two occasions). To be able to exclude organic sexual dysfunctions and other underlying illnesses, fasting blood glucose level, urinalysis, complete blood count, sex hormones, and prolactin levels were measured. All measurements were conducted by a single physician unaware of the treatment status. Moreover, all patients with erectile dysfunction (a reported IIEF-15-EFD score $\leq 21$ ) were excluded, as suggested by Salonia (16).

\section{Questionnaires and urological examinations}

Sexual health was evaluated using available validated instruments for the study of erectile function (International Index of Erectile Function-15 erectile function domain [IIEF-15-EFD]) (17) and ejaculatory status (PEDT) (18). In order to exclude all patients with lifelong $\mathrm{PE}$, a careful urological visit with disease history collection has been performed at the arrival at each Centre, as suggested by Jannini et al. (19). Moreover, the Male Sexual Health Questionnaire-Ejaculation Disorder (MSHQ-EjD) was also used to evaluate the ejaculation function (20).

\section{Assignment to the groups}

All patients were assigned to each group on the basis of the attending center. All patients attending to 5 centers (Rome 2 centers, Catania, Florence, Catanzaro) were assigned to Group A, while all patients attending another center (Trento) were assigned to Group B (controls). The study design is displayed in Figure 1.

\section{Group A}

The first group received one tablet orally a day for 3 months and one tablet consisted of $300 \mathrm{mg}$ of Satureya

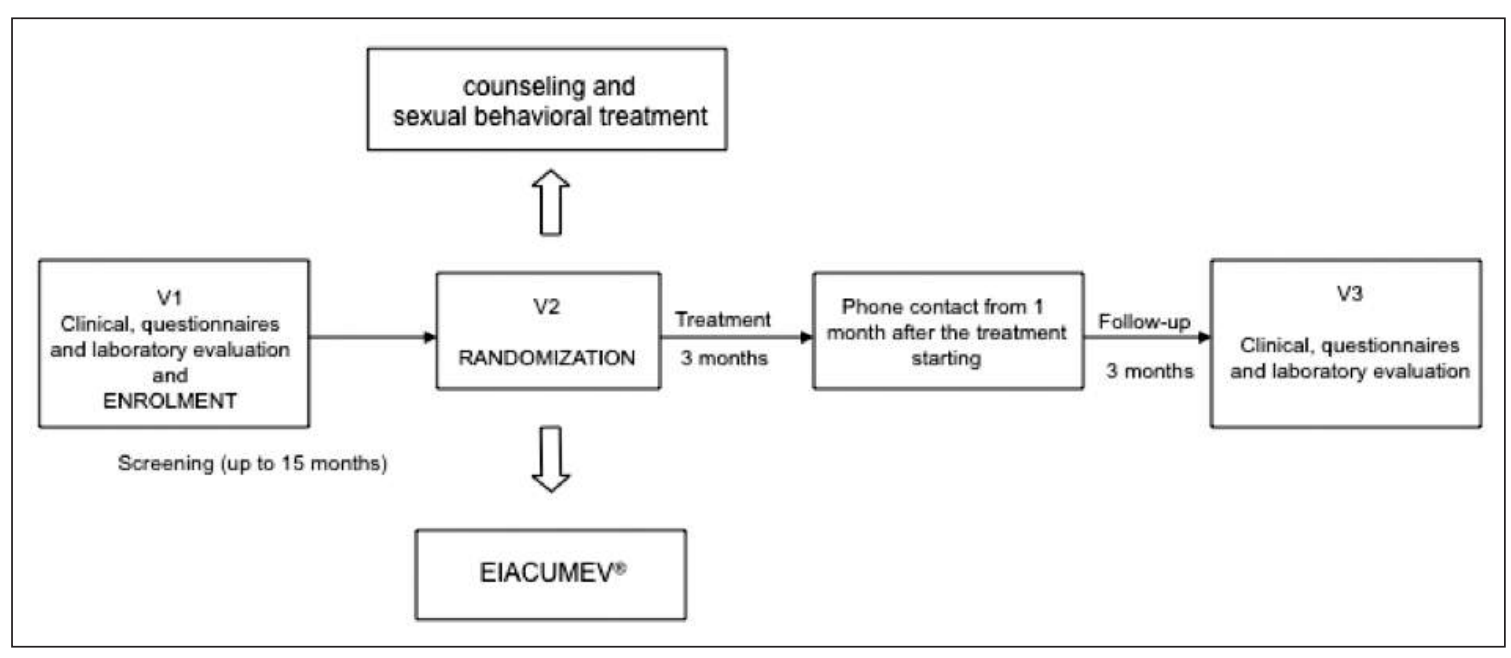

Figure 1. The figure shows the study design schedule. 


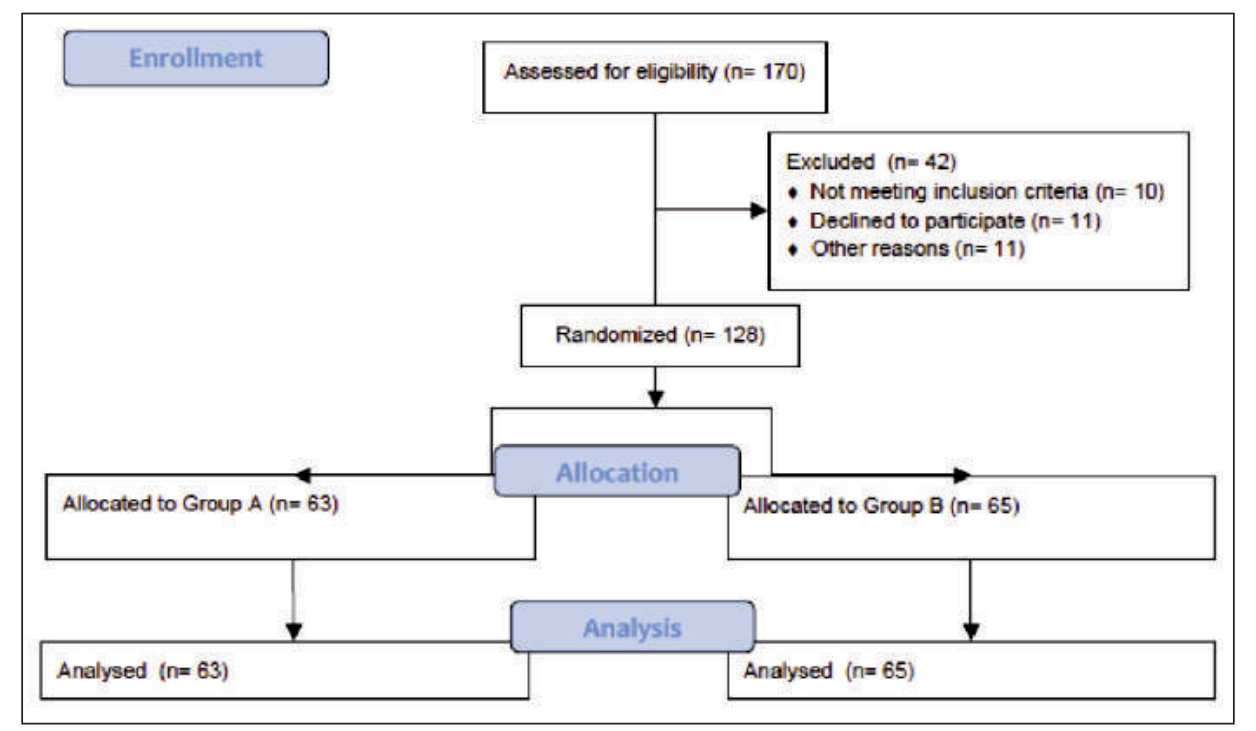

Figure 2.

The figure shows the study flow chart in accordance to the CONSORT guidelines. montana, Tribulus terrestris, Phyllanthus emblica, Cardamomo, L-tryptophan, ascorbic acid, vitamin PP, vitamin B1, vitamin B6 (EiacuMev ${ }^{\circledR}$, Farmaceutica MEV, Siena, Italy). We monitored adverse events on the light of common terminology criteria for adverse events (CTCAE) guidelines. Safety assessments included treatment-emergent adverse events (TEAEs), serious AEs (SAEs), and orthostatic vital signs (blood pressure and heart rate).

\section{Group B}

All patients in the control group underwent counseling and sexual behavioral treatment without any pharmacological compound. The counseling and sexual behavioral treatment has been carried out in collaboration with a dedicated psychologist, as commonly in our routinely clinical practice. The sexual counseling and psychotherapy has been performed in line with the suggestions of Rowland and Cooper (21).

\section{Main outcome measures}

The primary end point was stopwatch-measured IELT (held by the partner), reported for each intercourse episode. Secondary outcomes were the change from baseline to end point of MSHQ-EjD, IIEF-5 and PEDT.

\section{Statistical analysis and study population}

As null hypothesis, we assumed there would be no difference in terms of mean IELT score, between the two groups. A sample size of 60 subjects per treatment group provided $90 \%$ power (two-sided $\alpha=0.05$ ), to detect a 30 seconds difference in mean IELT between patients and controls. The maximum sample size was set to 70 subjects per group, allowing for a 15\% dropout rate. At baseline, the independent sample 2-tailed t-test was used to compare variables. For categorical parameters, chisquare test was applied. Changes from baseline to end of therapy were analyzed using ranked one-way analysis of variance (ANOVA) with a term for treatment group. Data were reported as means \pm standard deviation (SD). For all statistical comparisons, significance was considered as $\mathrm{p}<0.05$. All reported $\mathrm{p}$-values are two-sided. All statistical analyses were performed by using SPSS 11.0 for Apple-Macintosh (SPSS, Inc., Chicago, Illinois).

\section{RESULTS}

One hundred and seventy patients were enrolled. Fortytwo patients were excluded and 128 patients were finally randomized, 63 and 65 subjects in the group A and in the group B completed the study protocol. The flow chart of this study is presented in Figure 2.

\section{Baseline characteristics}

All anamnestic, clinical, and questionnaires characteristics of all enrolled patient are displayed in Table 1.

\section{Table 1.}

Baseline characteristics of patients enrolled.

\begin{tabular}{|c|c|c|}
\hline & Group A & Group B \\
\hline No. of patients & 63 & 65 \\
\hline Age (yr), mean $\pm S D$ & $32.03 \pm 8.68$ & $35.37 \pm 8.81$ \\
\hline $\mathrm{BMI}\left(\mathrm{Kg} / \mathrm{m}^{2}\right)$, mean $\pm \mathrm{SD}$ & $26.36 \pm 3.0$ & $25.2 \pm 3.5$ \\
\hline $\mathrm{LH}$, mean $\pm \mathrm{SD}$ & $87.96 \pm 56.92$ & $86.76 \pm 55.43$ \\
\hline FSH, mean \pm SD & $57.00 \pm 37.29$ & $54.61 \pm 35.70$ \\
\hline Total testosterone, mean & $6.89 \pm 3.87$ & $7.01 \pm 3.02$ \\
\hline Smoking habit, n (\%) & $25(39.68)$ & $26(36.92)$ \\
\hline IIEF-5, mean \pm SD & $27.31 \pm 2.86$ & $27.71 \pm 3.77$ \\
\hline PEDT, mean \pm SD & $13.82 \pm 2.79$ & $14.05 \pm 2.54$ \\
\hline IELT (sec), mean \pm SD & $54 \pm 17.76$ & $52 \pm 18.82$ \\
\hline EjQ, mean $\pm S D$ & $14.89 \pm 1.07$ & $15.01 \pm 1.35$ \\
\hline PSV, mean \pm SD & $31.52 \pm 6.60$ & $30.02 \pm 5.56$ \\
\hline EDV, mean $\pm S D$ & $1.5 \pm 1.0$ & $1.2 \pm 2.0$ \\
\hline \multicolumn{3}{|c|}{$\begin{array}{l}\text { BMI = Body Mass Index; IIEF-EF = International Index of Erectile Function-Erectile } \\
\text { Function; IIEF-IS = International Index of Erectile Function-Intercourse Satisfaction; IIEF } \\
\text { OF = International Index of Erectile Function-Orgasmic Function; IIEF-SD = International } \\
\text { Index of Erectile Function-Sexual Desire; IIEF-OS = International Index of Erectile } \\
\text { Function-Overall Satisfaction; MSHS-EJD = Male Sexual Health Questionnaire- } \\
\text { Ejaculation Disorder; SQoL-M = Sexual Quality of life instrument for Men; PSV = peak } \\
\text { systolic velocity; EDV = end diastolic velocity. }\end{array}$} \\
\hline
\end{tabular}


Table 2.

Mean changes from baseline to 3 months for primary and secondary outcomes.

\begin{tabular}{|lcc|}
\hline & Group A & Group B \\
\hline LH, mean \pm SD & $12.72 \pm 60.07$ & $10.50 \pm 55.00$ \\
\hline FSH, mean \pm SD & $-16.46 \pm 37.42^{a, b}$ & $2.52 \pm 5.76$ \\
\hline Total testosterone, mean & $1.55 \pm 10.93$ & $1.23 \pm 9.81$ \\
\hline IIEF-5, mean \pm SD & $1.04 \pm 1.67^{a}$ & $0.31 \pm 0.87$ \\
\hline PEDT, mean \pm SD & $-2.57 \pm 2.38^{a, b}$ & $-1.03 \pm 2.45$ \\
\hline IELT $($ sec $)$, mean \pm SD & $31.90 \pm 45.68^{a, b}$ & $1.60 \pm 9.81$ \\
\hline EjQ, mean $\pm S D$ & $-0.015 \pm 0.65$ & $0.02 \pm 0.89$ \\
\hline a $p<0.05$ vs. baseline; ${ }^{b} p<0.05$ vs. Group $B$. \\
\hline
\end{tabular}

\section{Follow-up examination}

The Table 2 lists the mean change differences from baseline to 3 months relative to main outcome measures. When concerning the primary endpoint of this study, we observed significant changes of the IIELT in the Group A (mean difference: $31.90 ; \mathrm{p}<0.05$ ) at 3 months and versus Group B at the intergroup analysis (mean difference: 30.30; $\mathrm{p}<0.05$ ). In the group $\mathrm{A}$, significant differences from baseline to last follow-up were observed relative to IIEF-5 (mean difference: 1.04; $\mathrm{p}<0.05$ ), PEDT (mean difference: $-2.57 ; \mathrm{p}<0.05$ ) and FSH (mean difference: $-16.46 ; \mathrm{p}<0.05)$.

Significant differences were found at the intergroup analysis when considering previous outcome measures (Table 2; Figure 3).

When considering serum TT and MSH-EjD, both groups did not show any difference after 3 months.

\section{Adverse events}

All subjects included in the study protocol, tolerated the treatments and none reported adverse events.

\section{Discussion}

Even if $\mathrm{PE}$ is one of the most common sexual dysfunctions among young male patients and in spite of the increasing interest in PE in the field of sexual medicine, a highly satisfactory treatment in terms of efficacy and safety is still needing (22). For these reasons, even if the preferred management of PE is on-demand Dapoxetine, many urologists used other treatment options in 35\% of the initial treatment cases and $50 \%$ of the second-line treatment cases (23). Here, we demonstrated that the use of tryptophan, Satureja montana, Tribulus terrestris, Phyllanthus emblica extracts is able to improve the patient's ejaculatory control, with a higher IELT and higher level of quality of life. Moreover, we demonstrated a high level of treatment compliance, probably due to the low frequency of adverse events and the efficacy of the treatment in terms of ejaculatory control improvement. The results of our study are probably due to synergic efficacy of Satureja montana and tryptophan. It is really dif-

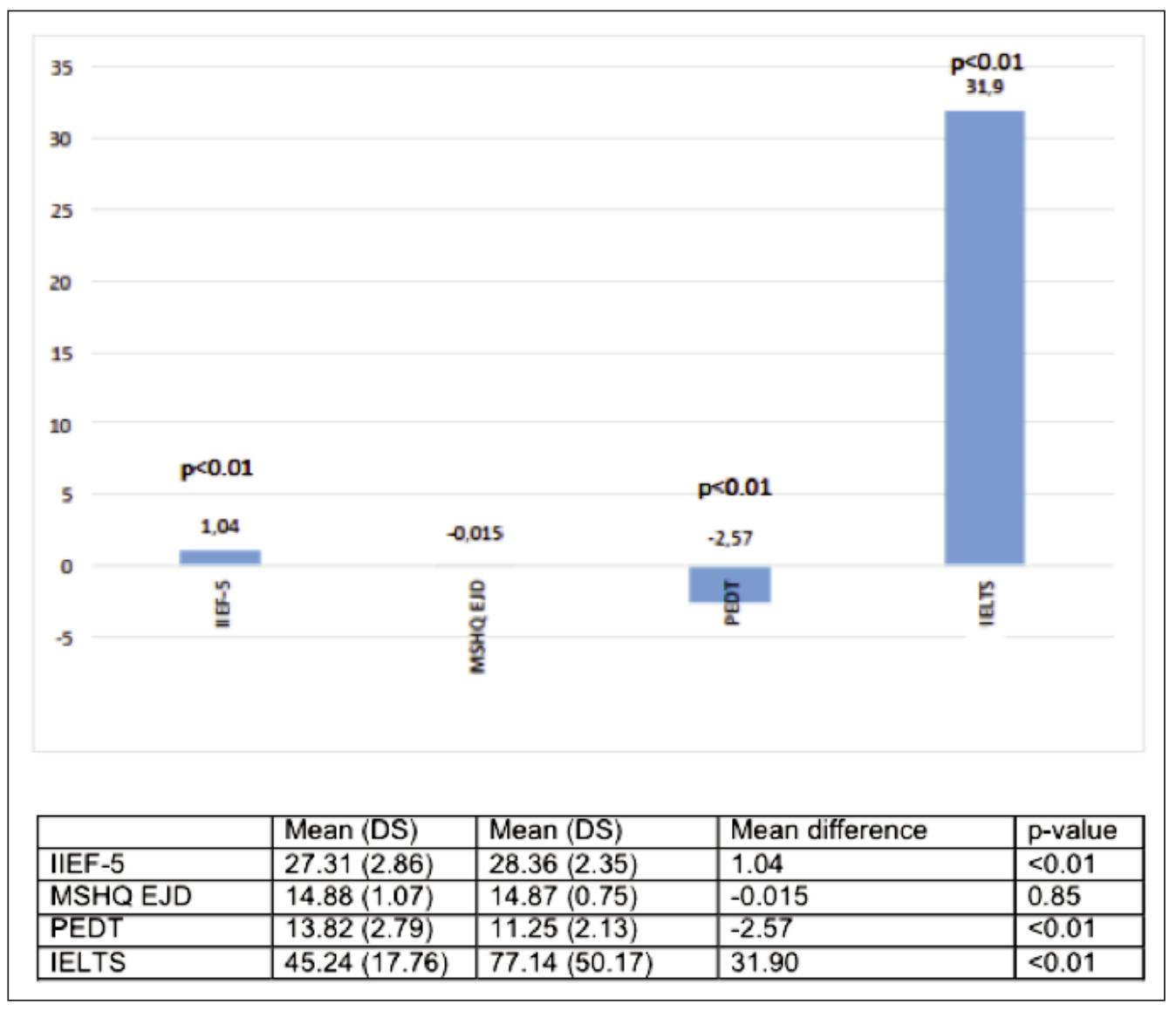

Figure 3.

The figure shows the significant differences found at the intergroup analysis (Group A) when considering outcome measures. VO: enrolment time; V1: follow-up visit (at 3 months from the treatment starting). IIEF-EF = International Index of Erectile Function-Erectile Function; MSHS-EJD = Male Sexual Health QuestionnaireEjaculation Disorder; $P E D T=$ premature ejaculation diagnostic tool; IELTS = Intravaginal Ejaculatory Latency Time: calculated as mean form partner and patient perceived IELT. 
ficult to evaluate the efficacy of each single compound on the PE improvement.

Several Authors demonstrated that serotonin may be associated with physical health as well as mood (24) and, in particular, in healthy people with high trait irritability, tryptophan, relative to placebo, decreased quarrelsome behaviors, increased agreeable behaviors and improved mood (25).

Nonpharmacologic methods of raising brain serotonin may not only improve mood, social functioning of healthy people, but would also improve sexual quality of life, improving the ejaculatory control.

5-hydroxytryptophan is the immediate precursor to serotonin and, by acting on 5-HT1A cerebral autoreceptors, spinal 5-HT1B and 5-HT2C receptors, has a determining role on ejaculation.

On the other hand, Zavatti et al. demonstrated that that the acute administration of Satureja montana extract is able to significantly increase the ejaculation latency and reduced the number of intromissions before ejaculation, without affecting the percentage of animals achieving ejaculation (100\% in all experimental groups) (13). They hypothesized that the efficacy of Satureja montana on premature ejaculation is due to its impact on male sexual behavior (13). However, even if the plants belonging to the genus Satureja are traditionally known as aphrodisiac remedy, no study was performed to confirm this property $(13,26)$. However, even if our results are promising, this study shows several limitations to take into account.

Firstly, the number of enrolled patients. Even if the number need to treat is correctly calculated is very important to highlight that the safety of phytotherapy should be evaluated with a long-term follow-up, in order to discover delayed adverse side effects. Moreover, the lack of a long-term follow-up in order to evaluate the long-term efficacy of this compound.

\section{Conclusion}

In our study, we demonstrated that patients affected by PE may significantly benefit from oral therapy with tryptophan, Satureja montana, Tribulus terrestris, Phyllanthus emblica extracts by improving IELT, PEDT and slightly IIEF-5.

\section{ACKNOWLEDGEMENTS}

We are grateful to Professor John Denton (Department of Modern Philology, University of Florence) for manuscript language revision.

\section{References}

1. Althof SE, McMahon CG, Waldinger MD, et al. An update of the International Society of Sexual Medicine's guidelines for the diagnosis and treatment of premature ejaculation (PE). J Sex Med. 2014; 11:1392-422.

2. Mirone V, Arcaniolo D, Rivas D, et al. PAUSE study team. Results from a prospective observational study of men with premature ejaculation treated with dapoxetine or alternative care: the PAUSE study. Eur Urol. 2014; 65:733-9.
3. McMahon CG. Dapoxetine: a new option in the medical management of premature ejaculation. Ther Adv Urol. 2012; 4:23351.

4. Corona G, Ricca V, Bandini E, et al. Selective serotonin reuptake inhibitor-induced sexual dysfunction. J Sex Med. 2009; 6:1259-69.

5. Fergusson D, Doucette S, Glass KC, et al. Association between suicide attempts and selective serotonin reuptake inhibitors: systematic review of randomized controlled trials. BMJ 2005; 330:396.

6. McMahon CG, Porst H. Oral agents for the treatment of premature ejaculation: review of efficacy and safety in the context of the recent International Society for Sexual Medicine criteria for lifelong premature ejaculation. J Sex Med. 2011; 8:2707-25.

7. Turner EH, Loftis JM, Blackwell AD. Serotonin a la carte: supplementation with the serotonin precursor 5-hydroxytryptophan. Pharmacol Ther 2006; 109:325-38.

8. Birdsall TC. 5-Hydroxytryptophan: a clinically-effective serotonin precursor. Altern Med Rev. 1998; 3:271-80.

9. McMahon C. Premature ejaculation: past, present, and future perspectives. J Sex Med. 2005; 2 Suppl 2:94-5.

10. Jannini EA, Porst H. Case studies. A practical approach to premature ejaculation. J Sex Med. 2011; 8 Suppl 4:360-7.

11. Magnussen I, Jensen TS, Rand JH, Van Woert MH. Plasma accumulation of metabolism of orally administered single dose L5-hydroxytryptophan in man. Acta Pharmacol Toxicol. 1981; 49:184-189.

12. Poldinger W, Calanchini B, Schwarz W. A functional-dimensional approach to depression: serotonin deficiency as a target syndrome in a comparison of 5-hydroxytryptophan and fluvoxamine. Psychopathology. 1991; 24:53-81.

13. Zavatti M, Zanoli P, Benelli A, et al. Experimental study on Satureja montana as a treatment for premature ejaculation. J Ethnopharmacol. 2011; 133:629-33.

14. Goswami SK, Pandre MK, Jamwal R, et al. Screening for Rhokinase 2 inhibitory potential of Indian medicinal plants used in management of erectile dysfunction. J Ethnopharmacol. 2012; 144:483-9.

15. Hee Ju Kim, Joonki Kim, Ki Sung Kang, et al. Neuroprotective Effect of Chebulagic Acid via Autophagy Induction in SH-SY5Y Cells. Biomol Ther (Seoul) 2014; 22:275-281.

16. Salonia A, Maga T, Colombo R, et al. A prospective study comparing paroxetine alone versus paroxetine plus sildenafil in patients with premature ejaculation. J Urol. 2002; 168:2486-9.

17. Cappelleri JC, Rosen RC, Smith MD, et al. Diagnostic evaluation of the erectile function domain of the International Index of Erectile Function. Urology. 1999; 54:346-51.

18. Symonds T, Perelman MA, Althof S, et al. Development and validation of a premature ejaculation diagnostic tool. Eur Urol. 2007; 52:565-73.

19. Jannini EA, Maggi M, Lenzi A. Evaluation of premature ejaculation. J Sex Med. 2011; 8 Suppl 4:328-34.

20. Rosen RC, Catania J, Pollack L, et al. Male Sexual Health Questionnaire (MSHQ): scale development and psychometric validation. Urology. 2004; 64:777-82.

21. Rowland D, Cooper S. Practical tips for sexual counseling and psychotherapy in premature ejaculation. J Sex Med. 2011; 8 Suppl 4:342-52. 
22. Yang DY, Ko K, Lee WK, et al. Urologist's Practice Patterns Including Surgical Treatment in the Management of Premature Ejaculation: A Korean Nationwide Survey. World J Mens Health. 2013; 31:226-31.

23. Shindel A, Nelson C, Brandes S. Urologist practice patterns in the management of premature ejaculation: a nationwide survey. $J$ Sex Med. 2008; 5:199-205.
24. Young SN. How to increase serotonin in the human brain without drugs. J Psychiatry Neurosci. 2007; 32:394-399.

25. aan het Rot M, Moskowitz DS, Pinard G, et al. Social behaviour and mood in everyday life: effects of tryptophan in quarrelsome individuals. J Psychiatry Neurosci. 2006; 31:253-62.

26. Haeri S, Minaie B, Amin G, et al. Effect of Satureja khuzestanica essential oil on male rat fertility. Fitoterapia 2006; 77:495-499.

\section{Correspondence}

Salvatore Sansalone, MD

salvatore.sansalone@yahoo.it

Department of Experimental Medicine and Surgery, University Tor Vergata,

Viale Oxford, 81 - 00133 Rome, Italy

Giorgio Ivan Russo, MD

Department of Urology, University of Catania, Catania, Italy

Nicola Mondaini, $M D$

Urologic Unit, Santa Maria Annunziata Hospital, Bagno a Ripoli, Florence, Italy

Francesco Cantiello, MD

Urology Unit, Magna Graecia University of Catanzaro, Italy

Gabriele Antonini, MD

Department of Urology, "Sapienza" Rome University, Rome, Italy

Tommaso Cai, MD

Department of Urology, Santa Chiara Regional Hospital, Trento, Italy 